\title{
ON UPPER AND LOWER ALMOST
}

\section{CONTRA-I-CONTINUOUS MULTIFUNCTIONS}

\author{
C. Arivazhai ${ }^{1}$, N. Rajesh ${ }^{2}$, S. Shanthi ${ }^{3}$ \\ ${ }^{1,2}$ Department of Mathematics \\ Rajah Serfoji Govt. College \\ Thanjavur, 613005, Tamilnadu, INDIA \\ ${ }^{3}$ Department of Mathematics \\ Arignar Anna Govt. Arts College \\ Namakkal, 637 001, Tamilnadu, INDIA
}

\begin{abstract}
In this paper, we introduce and study the almost contra-I-continuous multifunctions between topological spaces and obtain some characterizations and properties of such multifunctions.
\end{abstract}

AMS Subject Classification: 54C60, 54C08

Key Words: regular open set, $\mathcal{I}$-open set, almost contra- $\mathcal{I}$-continuous multifunctions

\section{Introduction}

It is well known that various types of functions play a significant role in the theory of classical point set topology. A great number of papers dealing with such functions have appeared, and a good number of them have been extended to the setting of multifunctions $[2,6,22,23,24,25]$. This implies that both, functions and multifunctions are important tools for studying other properties of spaces and for constructing new spaces from previously existing ones. The concept of ideals in topological spaces has been introduced and studied by Kuratowski [13] and Vaidyanathaswamy, [29]. An ideal $\mathcal{I}$ on a topological space

Received: $\quad$ February 3, 2017

Revised: July 16, 2017

Published: $\quad$ August 8, 2017

$\S_{\text {Correspondence author }}$ (c) 2017 Academic Publications, Ltd. url: www.acadpubl.eu 
$(X, \tau)$ is a nonempty collection of subsets of $X$ which satisfies (i) $A \in \mathcal{I}$ and $B \subset A$ implies $B \in \mathcal{I}$ and (ii) $A \in \mathcal{I}$ and $B \in \mathcal{I}$ implies $A \cup B \in \mathcal{I}$. Given a topological space $(X, \tau)$ with an ideal $\mathcal{I}$ on $X$ and if $\mathcal{P}(X)$ is the set of all subsets of $X$, a set operator $(.)^{\star}: \mathcal{P}(X) \rightarrow \mathcal{P}(X)$, called the local function [29] of $A$ with respect to $\tau$ and $\mathcal{I}$, is defined as follows: for $A \subset X, A^{\star}(\tau, \mathcal{I})=$ $\{x \in X \mid U \cap A \notin \mathcal{I}$ for every $U \in \tau(x)\}$, where $\tau(x)=\{U \in \tau \mid x \in U\}$. A Kuratowski closure operator $\mathrm{Cl}^{\star}($.$) for a topology \tau^{\star}(\tau, \mathcal{I})$ called the $*$-topology, finer than $\tau$ is defined by $\mathrm{Cl}^{\star}(A)=A \cup A^{\star}(\tau, \mathcal{I})$ when there is no chance of confusion, $A^{\star}(\mathcal{I})$ is denoted by $A^{\star}$. If $\mathcal{I}$ is an ideal on $X$, then $(X, \tau, \mathcal{I})$ is called an ideal topological space. In 1990, Jankovic and Hamlett [10] introduced the notion of $\mathcal{I}$-open sets in topological spaces. In 1992, Abd El-Monsef et al. [1] further investigated $\mathcal{I}$-open sets and $\mathcal{I}$-continuous functions. Several characterizations and properties of $\mathcal{I}$-open sets were provided in [1, 16]. Recently, Akdag [2] introduced and studied the concept of $\mathcal{I}$-continuous multifunctions in topological spaces. In this paper, we introduce and study almost contra- $\mathcal{I}$-continuous multifunctions between topological spaces and obtain some characterizations of such multifunctions.

\section{Preliminaries}

Throughout this paper, $(X, \tau)$ and $(Y, \sigma)$ (or simply $X$ and $Y$ ) always mean topological spaces in which no separation axioms are assumed unless explicitly stated. Let $A$ be a subset of a space $X$. For a subset $A$ of $(X, \tau), \operatorname{Cl}(A)$ and $\operatorname{Int}(A)$ denote the closure of $A$ with respect to $\tau$ and the interior of $A$ with respect to $\tau$, respectively. A subset $S$ of an ideal topological space $(X, \tau, \mathcal{I})$ is $\mathcal{I}$-open [10] if $S \subset \operatorname{Int}\left(S^{*}\right)$. The complement of an $\mathcal{I}$-closed set is said to be an $\mathcal{I}$-open set. The $\mathcal{I}$-closure and the $\mathcal{I}$-interior, that can be defined in the same way as $\operatorname{Cl}(A)$ and $\operatorname{Int}(A)$, respectively, will be denoted by $\mathcal{I} \mathrm{Cl}(A)$ and $\mathcal{I} \operatorname{Int}(A)$, respectively. The family of all $\mathcal{I}$-open (resp. $\mathcal{I}$-closed) sets of $(X, \tau, \mathcal{I})$ is denoted by $\mathcal{I} O(X)(\operatorname{resp} . \mathcal{I} C(X))$. The family of all $\mathcal{I}$-open (resp. $\mathcal{I}$-closed) sets of $(X, \tau, \mathcal{I})$ containing a point $x \in X$ is denoted by $\mathcal{I} O(X, x)$ (resp. $\mathcal{I} C(X, x)$ ). A subset $A$ is said to be regular open [27] (resp. semiopen [14], preopen [15], $\alpha$-open [18], semi-preopen [3]) if $A=\operatorname{Int}(\mathrm{Cl}(A))$ (resp. $A \subset$ $\mathrm{Cl}(\operatorname{Int}(A)), A \subset \operatorname{Int}(\mathrm{Cl}(A)), A \subset \operatorname{Int}(\mathrm{Cl}(\operatorname{Int}(A))), A \subset \mathrm{Cl}(\operatorname{Int}(\mathrm{Cl}(A))))$. The complement of a regular open (resp. semiopen, preopen, semi-preopen) set is called a regular closed (resp. semiclosed, preclosed, semi-preclosed). The intersection of all semiclosed (resp. preclosed, $\alpha$-closed, semi-preclosed) subsets of $(X, \tau)$ containing $A \subset X$ is called the semiclosure (resp. preclosure, $\alpha$ - 
closure, semi-preclosure) of $A$ and is denoted by $s \mathrm{Cl}(A)$ (resp. $p \mathrm{Cl}(A), \alpha \mathrm{Cl}(A)$, $s p \mathrm{Cl}(A))$. The $\theta$-semiclosure [11] of $A$, denoted by $s \mathrm{Cl}_{\theta}(A)$, is defined to be the set of all $x \in X$ such that $A \cap \mathrm{Cl}(U) \neq \emptyset$ for every semiopen set $U$ containing $x$. A subset $A$ is called $\theta$-semiclosed [11] if and only if $A=$ $s \mathrm{Cl}_{\theta}(A)$. The complement of a $\theta$-semiclosed set is called a $\theta$-semiopen set [11]. The family of all regular open (resp. regular closed, semiopen, semiclosed, $\alpha$ open, semi-preopen, semi-preclosed) sets of $(X, \tau)$ is denoted by $R O(X)$ (resp. $R C(X), S O(X), S C(X), \alpha O(X), S P O(X), S P C(X))$. By a multifunction $F:(X, \tau, \mathcal{I}) \rightarrow(Y, \sigma)$, following [7], we shall denote the upper and lower inverse of a set $B$ of $Y$ by $F^{+}(B)$ and $F^{-}(B)$, respectively, that is, $F^{+}(B)=$ $\{x \in X: F(x) \subset B\}$ and $F^{-}(B)=\{x \in X: F(x) \cap B \neq \emptyset\}$. In particular, $F^{-}(Y)=\{x \in X: y \in F(x)\}$ for each point $y \in Y$ and for each $A \subset X, F(A)$ $=\cup_{x \in A} F(x)$. Then $F$ is said to be surjection if $F(X)=Y$ and injection if $x \neq y$ implies $F(x) \cap F(y)=\emptyset$.

Definition 2.1. A multifunction $F:(X, \tau, \mathcal{I}) \rightarrow(Y, \sigma)$ is said to be:

1. upper almost $\mathcal{I}$-continuous [4] if for each point $x \in X$ and each open set $V$ containing $F(x)$, there exists $U \in \mathcal{I} O(X, x)$ such that $U \subset F^{+}(\operatorname{Int}(\mathrm{Cl}(V)))$;

2. lower almost $\mathcal{I}$-continuous [4] if for each point $x \in X$ and each open set $V$ such that $F(x) \cap V \neq \emptyset$, there exists $U \in \mathcal{I} O(X, x)$ such that $U \subset F^{-}(\operatorname{Int}(\mathrm{Cl}(V)))$.

3. upper contra- $\mathcal{I}$-continuous [5] if for each point $x \in X$ and each closed set $V$ containing $F(x)$, there exists $U \in \mathcal{I} O(X, x)$ such that $U \subset F^{+}(V)$;

4. lower contra- $\mathcal{I}$-continuous [5] if for each point $x \in X$ and each closed set $V$ such that $F(x) \cap V \neq \emptyset$, there exists $U \in \mathcal{I} O(X, x)$ such that $U \subset F^{-}(V)$;

5. upper weakly $\mathcal{I}$-continuous [6] if for each $x \in X$ and each open set $V$ of $Y$ such that $x \in F^{+}(V)$, there exists $U \in \mathcal{I} O(X, x)$ such that $U \subset$ $F^{+}(\mathrm{Cl}(V))$;

6. lower weakly $\mathcal{I}$-continuous [6] if for each $x \in X$ and each open set $V$ of $Y$ such that $x \in F^{-}(V)$, there exists $U \in \mathcal{I} O(X, x)$ such that $U \subset$ $F^{-}(\mathrm{Cl}(V))$.

Lemma 2.2. [1] Let $A$ and $B$ be subsets of a space $(X, \tau)$.

1. If $A \in \mathcal{I} O(X)$ and $B \in \tau$, then $A \cap B \in \mathcal{I} O(B)$;

2. If $A \in \mathcal{I} O(B)$ and $B \in \mathcal{I} O(X)$, then $A \in \mathcal{I} O(X)$. 
Lemma 2.3. [20] For a multifunction $F:(X, \tau) \rightarrow(Y, \sigma)$, the following holds:

1. $G_{F}^{+}(A \times B)=A \cap F^{+}(B)$;

2. $G_{F}^{-}(A \times B)=A \cap F^{-}(B)$,

for any subset $A$ of $X$ and $B$ of $Y$.

\section{On Upper and Lower Almost Contra-I-Continuous Multifunctions}

Definition 3.1. A multifunction $F:(X, \tau, \mathcal{I}) \rightarrow(Y, \sigma)$ is said to be:

1. upper almost contra-I-continuous if for each point $x \in X$ and each regular closed set $V$ with $x \in F^{+}(V)$, there exists $U \in \mathcal{I} O(X, x)$ such that $U \subset F^{+}(V)$;

2. lower almost contra- $\mathcal{I}$-continuous if for each point $x \in X$ and each regular closed set $V$ with $x \in F^{-}(V)$, there exists $U \in \mathcal{I} O(X, x)$ such that $U \subset F^{-}(V)$.

Theorem 3.2. If $F:(X, \tau, \mathcal{I}) \rightarrow(Y, \sigma)$ is an upper (lower) almost contra$\mathcal{I}$-continuous multifunction, then it is upper (lower) weakly $\mathcal{I}$-continuous.

Proof. Let $x \in X$ and $V$ be an open subset of $Y$ with $F(x) \subset V$. This implies that $\mathrm{Cl}(V)$ is a regular closed set with $F(x) \subset \mathrm{Cl}(V)$. Since $F$ is upper almost contra- $\mathcal{I}$-continuous, there exists $U \in \mathcal{I} O(X, x)$ such that $U \subset$ $F^{+}(\mathrm{Cl}(V))$. Hence, $F$ is upper weakly $\mathcal{I}$-continuous

The following example shows that the converse of the above Theorem 3.2 is not true in general.

Example 3.3. Let $X=\{a, b, c, d\}, \tau=\{\emptyset, X,\{a\},\{c\},\{a, b\},\{a, c\}$, $\{a, b, c\},\{a, c, d\}\}$ and $\mathcal{I}=\{\emptyset\}$. Define a multifunction $F:(X, \tau, \mathcal{I}) \rightarrow(X, \tau)$ by $F(a)=\{b\}, F(b)=\{b\}, F(c)=\{a\}, F(d)=\{d\}$. Then $F$ is upper weakly $\mathcal{I}$-continuous but it is not upper almost contra- $\mathcal{I}$-continuous.

Corollary 3.4. If $F:(X, \tau, \mathcal{I}) \rightarrow(Y, \sigma)$ is almost contra- $\mathcal{I}$-continuous, then it is weakly $\mathcal{I}$-continuous.

Theorem 3.5. If $F:(X, \tau, \mathcal{I}) \rightarrow(Y, \sigma)$ is an upper (lower) contra- $\mathcal{I}$ continuous multifunction, then it is upper (lower) almost contra $\mathcal{I}$-continuous multifunction. 
Proof. The proof is obvious.

The following example shows that the converse of the above Theorem 3.5 is not true in general.

Example 3.6. Let $X=\Re$ with the usual topology and $\mathcal{I}=\{\emptyset\}$. Then the multifunction $F:(\Re, \tau, \mathcal{I}) \rightarrow(\Re, \tau)$ defined by $F(x)=\{x\}$ is upper almost contra- $\mathcal{I}$-continuous multifunction but is not upper contra $\mathcal{I}$-continuous multifunction.

Corollary 3.7. If $F:(X, \tau, \mathcal{I}) \rightarrow(Y, \sigma)$ is contra- $\mathcal{I}$-continuous, then it is almost contra- $\mathcal{I}$-continuous.

Theorem 3.8. For a multifunction $F:(X, \tau, \mathcal{I}) \rightarrow(Y, \sigma)$, the following statements are equivalent:

1. $F$ is upper almost contra-I-continuous;

2. $F^{+}(A) \in \mathcal{I} O(X)$ for every regular closed $A$ of $Y$;

3. $F^{-}(U) \in \mathcal{I} C(X)$ for every regular open subset $U$ of $Y$;

4. $F^{-}(\operatorname{Int}(\mathrm{Cl}(A))) \in \mathcal{I} C(X)$ for every open subset $V$ of $Y$;

5. $F^{+}(\mathrm{Cl}(\operatorname{Int}(A))) \in \mathcal{I} O(X)$ for every closed subset $V$ of $Y$;

6. for each $x \in X$ and for each $V \in S O(Y)$ with $F(x) \subset V$, there exists $U \in \mathcal{I} O(X, x)$ such that $F(U) \subset \mathrm{Cl}(V)$;

7. $F^{+}(V) \subset \mathcal{I} \operatorname{Int}\left(F^{+}(\mathrm{Cl}(V))\right)$ for every $V \in S O(Y)$.

Proof. $(1) \Leftrightarrow(2)$ : Let $A \in R C(Y)$ and $x \in F^{+}(A)$. Since $F$ is upper almost contra- $\mathcal{I}$-continuous, there exists $U \in \mathcal{I} O(X, x)$ such that $U \subset F^{+}(A)$. Thus, $F^{+}(A) \in \mathcal{I} O(X)$. The converse is obvious.

$(2) \Leftrightarrow(3)$ and $(4) \Leftrightarrow(5)$ : It follows from the fact that $F^{+}(Y \backslash A)=$ $X \backslash F^{-}(A)$ for every subset $A$ of $Y$.

$(3) \Leftrightarrow(4)$ : Let $A$ be an open subset of $Y$. Since $\operatorname{Int} \mathrm{Cl}(A))$ is regular open, then $F^{-}(\operatorname{Int}(\mathrm{Cl}(A)))$ is $\mathcal{I}$-closed. The converse is obvious. $(5) \Leftrightarrow(2)$ : It is similar to that of $(3) \Leftrightarrow(4)$.

$(6) \Rightarrow(7)$ : Let $V \in S O(Y)$ and $x \in F^{+}(V)$. Then $F(x) \subset V$. By (6), there exists $U \in \mathcal{I} O(X, x)$ such that $F(U) \subset \mathrm{Cl}(V)$. This implies that $x \in U \subset$ $F^{+}(\mathrm{Cl}(V))$. Hence, $x \in \mathcal{I} \operatorname{Int}\left(F^{+}(\mathrm{Cl}(V))\right)$ and $F^{+}(V) \subset \mathcal{I} \operatorname{Int}\left(F^{+}(V)\right)$.

$(7) \Rightarrow(2)$ : Let $A \in R C(Y)$. Since $A \in S O(Y)$, then

$$
F^{+}(A) \subset \mathcal{I} \operatorname{Int}\left(F^{+}(\mathrm{Cl}(A))\right) ;
$$


hence $F^{+}(A) \in \mathcal{I} O(X)$.

$(2) \Rightarrow(6)$ : Let $x \in X$ and $V \in S O(Y)$ with $F(x) \subset V$. Since $\mathrm{Cl}(V) \in$ $R C(Y)$, there exists $A \in \mathcal{I} O(X, x)$ such that $A \subset F^{+}(\mathrm{Cl}(V))$. Hence $F(A) \subset$ $\mathrm{Cl}(V)$.

Theorem 3.9. For a multifunction $F:(X, \tau, \mathcal{I}) \rightarrow(Y, \sigma)$, the following statements are equivalent:

1. $F$ is lower almost contra- $\mathcal{I}$-continuous;

2. $F^{-}(A) \in \mathcal{I} O(X)$ for every regular closed $A$ of $Y$;

3. $F^{+}(U) \in \mathcal{I} C(X)$ for every regular open subset $U$ of $Y$;

4. $F^{+}(\operatorname{Int}(\mathrm{Cl}(A))) \in \mathcal{I} C(X)$ for every open subset $V$ of $Y$;

5. $F^{-}(\mathrm{Cl}(\operatorname{Int}(A))) \in \mathcal{I} O(X)$ for every closed subset $V$ of $Y$;

6. for each $x \in X$ and for each $V \in S O(Y)$ with $F(x) \cap V \neq \emptyset$, there exists $U \in \mathcal{I} O(X, x)$ such that $F(u) \cap \mathrm{Cl}(V) \neq \emptyset$ for each $u \in U$;

7. $F^{-}(V) \subset \mathcal{I} \operatorname{Int}\left(F^{-}(\mathrm{Cl}(V))\right)$ for every $V \in S O(Y)$.

Proof. The proof is similar to that of Theorem 3.8.

Theorem 3.10. For a multifunction $F:(X, \tau, \mathcal{I}) \rightarrow(Y, \sigma)$, the following statements are equivalent:

1. $F$ is lower almost contra-I-continuous;

2. $F^{-}(A) \in \mathcal{I} O(X)$ for every $\theta$-semiopen $A$ of $Y$;

3. $F^{+}(U) \in \mathcal{I} C(X)$ for every $\theta$-semiclosed subset $U$ of $Y$;

4. $\mathcal{I} \mathrm{Cl}\left(F^{+}(\operatorname{Int}(\mathrm{Cl}(B)))\right) \subset F^{+}(s \mathrm{Cl}(B)$ for every subset $B$ of $Y$;

5. $\mathcal{I} \mathrm{Cl}\left(F^{+}(B)\right) \subset F^{+}\left(s \mathrm{Cl}_{\theta}(B)\right)$ for every subset $B$ of $Y$;

6. $F(\mathcal{I} \mathrm{Cl}(A)) \subset s \mathrm{Cl}_{\theta}(F(A))$ for every subset $A$ of $X$.

Proof. $(1) \Rightarrow(2)$ : Let $G$ be any $\theta$-semiopen set of $Y$. There exists a family of regular closed sets $\left\{K_{\alpha}: \alpha \in \Delta\right\}$ such that $G=\cup\left\{K_{\alpha}: \alpha \in \Delta\right\}$. It follows from Theorem 3.5 (ii) that $F^{-}(G)=\cup\left\{F^{-}\left(K_{\alpha}\right): \alpha \in \Delta\right\}$ is $\mathcal{I}$-open.

$(2) \Rightarrow(3)$ : This is obvious. 
$(3) \Rightarrow(4)$ : Let $B$ be any subset of $Y$. Then $\operatorname{Int}(\mathrm{Cl}(B))$ is regular open and it is $\theta$-semiclosed in $Y$. Therefore, we have that $F^{+}(\operatorname{Int}(\mathrm{Cl}(B)))$ is $\mathcal{I}$-closed and $\mathcal{I} \mathrm{Cl}\left(F^{+}(\operatorname{Int}(\mathrm{Cl}(B)))\right)=F^{+}(\operatorname{Int}(\mathrm{Cl}(B))) \subset F^{+}(s \mathrm{Cl}(B))$.

$(4) \Rightarrow(5)$ : Let $B$ be any subset of $Y$. For any regular open set $V$ with $B \subset V$, we have $\mathcal{I} \mathrm{Cl}\left(F^{+}(B)\right) \subset \mathrm{Cl}\left(F^{+}(V)\right)=\mathcal{I} \mathrm{Cl}\left(F^{+}(\operatorname{Int}(\mathrm{Cl}(V)))\right) \subset$ $F^{+}(s \mathrm{Cl}(V))=F^{+}(V)$. Therefore, $\mathcal{I} \mathrm{Cl}\left(F^{+}(B)\right) \subset F^{+}(\cap\{V \in R O(Y): B \subset$ $V\})=F^{+}\left(s \mathrm{Cl}_{\theta}(B)\right)$.

$(5) \Rightarrow(1)$ : Let $V$ be any semiopen set of $Y$. Then we have

$$
\begin{aligned}
& X \backslash \mathcal{I} \operatorname{Int}\left(F^{-}(\mathrm{Cl}(V))\right) \\
& \qquad \begin{aligned}
\mathcal{I} \mathrm{Cl}\left(F^{+}(Y \backslash \mathrm{Cl}(V))\right) \subset F^{+}\left(s \mathrm{Cl}_{\theta}(Y \backslash \mathrm{Cl}(V))\right)=F^{+}(Y \backslash \mathrm{Cl}(V)) \\
=X \backslash F^{-}(\mathrm{Cl}(V)) .
\end{aligned}
\end{aligned}
$$

Therefore, we obtain $F^{-}(V) \subset F^{-}(\mathrm{Cl}(V)) \subset \mathcal{I} \operatorname{Int}\left(F^{-}(\mathrm{Cl}(V))\right)$. By Theorem $3.9(7), F$ is lower almost contra- $\mathcal{I}$-continuous.

$(5) \Rightarrow(6)$ : Let $A$ be a subset of $X$ and $B=F(A)$. Then $A \subset F^{+}(B)$ and $\mathcal{I} \mathrm{Cl}(A) \subset \mathcal{I C l}\left(F^{+}(B)\right) \subset F^{+}\left(s \mathrm{Cl}_{\theta}(B)\right)$. Therefore, we have $F(\mathcal{I} \mathrm{Cl}(A)) \subset$ $F\left(F^{+}\left(s \mathrm{Cl}_{\theta}(B)\right)\right) \subset s \mathrm{Cl}_{\theta}(B)=s \mathrm{Cl}_{\theta}(F(A))$.

$(6) \Rightarrow(5)$ : Let $B$ be any subset of $Y$. Then we have $F\left(\mathcal{I} \mathrm{Cl}\left(F^{+}(B)\right)\right) \subset$ $s \mathrm{Cl}_{\theta}\left(F\left(F^{+}(B)\right)\right) \subset s \mathrm{Cl}_{\theta}(B)$; hence $\mathcal{I} \mathrm{Cl}\left(F^{+}(B)\right) \subset F^{+}\left(s \mathrm{Cl}_{\theta}(B)\right)$.

Theorem 3.11. For a multifunction $F:(X, \tau, \mathcal{I}) \rightarrow(Y, \sigma)$, the following statements are equivalent:

1. $F$ is upper almost contra- $\mathcal{I}$-continuous;

2. $\mathcal{I} \mathrm{Cl}\left(F^{-}(\operatorname{Int}(K))\right) \subset F^{-1}(K)$ for every semiclosed set $K$ of $Y$;

3. $\mathcal{I} \mathrm{Cl}\left(F^{-}(\operatorname{Int}(s \mathrm{Cl}(B)))\right) \subset F^{+}(s \mathrm{Cl}(B))$ for every subset $B$ of $Y$;

4. $F^{+}(s \operatorname{Int}(B)) \subset \mathcal{I} \operatorname{Int}\left(F^{+}(\mathrm{Cl}(s \operatorname{Int}(B)))\right)$ for every subset $B$ of $Y$.

Proof. $(1) \Rightarrow(2)$ : Let $K$ be a semiclosed set of $Y$. Then $Y \backslash K$ is semiopen. By Theorem $3.8(7)$, it follows that $F^{+}(Y \backslash K) \subset \mathcal{I} \operatorname{Int}\left(F^{+}(Y \backslash \operatorname{Int}(K))\right)$. Hence

$$
\begin{aligned}
X \backslash F^{-}(K) \subset \mathcal{I} \operatorname{Int}\left(F^{+}(Y \backslash\right. & \operatorname{Int}(K))) \\
& =\mathcal{I} \operatorname{Int}\left(X \backslash F^{-}(\operatorname{Int}(K))\right)=X \backslash \mathcal{I} \operatorname{Cl}\left(F^{-}(\operatorname{Int}(K))\right) .
\end{aligned}
$$

Hence, $\mathcal{I} \mathrm{Cl}\left(F^{-}(\operatorname{Int}(K))\right) \subset F^{-1}(K)$.

$(2) \Rightarrow(3)$ : Let $B$ be any subset of $Y$. Then $s \mathrm{Cl}(B)$ is semiclosed in $Y$ and hence $\mathcal{I} \mathrm{Cl}\left(F^{-}(\operatorname{Int}(s \mathrm{Cl}(B)))\right) \subset F^{-}(s \mathrm{Cl}(B))$. 
$(3) \Rightarrow(4)$ : Let $B$ be any subset of $Y$. Then we have

$$
\begin{array}{r}
X \backslash F^{+}(s \operatorname{Int}(B))=F^{-}(s \mathrm{Cl}(Y \backslash B)) \supset \mathcal{I} \mathrm{Cl}\left(F^{-}(\operatorname{Int}(s \mathrm{Cl}(Y \backslash B)))\right) \\
=\mathcal{I} \mathrm{Cl}\left(F^{-}(\operatorname{Int}(Y \backslash s \operatorname{Int}(B)))\right)=\mathcal{I} \mathrm{Cl}\left(F^{-}(Y \backslash \mathrm{Cl}(s \operatorname{Int}(B)))\right) \\
=\mathcal{I} \mathrm{Cl}\left(X \backslash F^{+}(\mathrm{Cl}(s \operatorname{Int}(B)))\right)=X \backslash \mathcal{I} \operatorname{Int}\left(F^{+}(\mathrm{Cl}(s \operatorname{Int}(B)))\right) .
\end{array}
$$

Hence, $F^{+}(s \operatorname{Int}(B)) \subset \mathcal{I} \operatorname{Int}\left(F^{+}(\mathrm{Cl}(s \operatorname{Int}(B)))\right)$.

$(4) \Rightarrow(1)$ : Let $V$ be any semiopen set of $Y$. Then $V=s \operatorname{Int}(V)$ and hence $F^{+}(V) \subset \mathcal{I} \mathrm{Cl}\left(F^{+}(\mathrm{Cl}(V))\right)$. By Theorem $3.8(7), F$ is upper almost contra- $\mathcal{I}$ continuous.

Theorem 3.12. For a multifunction $F:(X, \tau, \mathcal{I}) \rightarrow(Y, \sigma)$, the following statements are equivalent:

1. $F$ is lower almost contra- $\mathcal{I}$-continuous;

2. $\mathcal{I} \mathrm{Cl}\left(F^{+}(\operatorname{Int}(K))\right) \subset F^{+}(K)$ for every semiclosed set $K$ of $Y$;

3. $\mathcal{I} \mathrm{Cl}\left(F^{+}(\operatorname{Int}(s \mathrm{Cl}(B)))\right) \subset F^{+}(s \mathrm{Cl}(B))$ for every subset $B$ of $Y$;

4. $F^{-}(s \operatorname{Int}(B)) \subset \mathcal{I} \operatorname{Int}\left(F^{-}(\mathrm{Cl}(s \operatorname{Int}(B)))\right)$ for every subset $B$ of $Y$;

Proof. The proof is similar to that of Theorem 3.11.

Recall that a topological space is said to be extremally disconnected if the closure of every open set is open in the space.

Theorem 3.13. Let $(Y, \sigma)$ be a extremally disconnected space. Then a multifunction $F:(X, \tau, \mathcal{I}) \rightarrow(Y, \sigma)$ is upper almost contra- $\mathcal{I}$-continuous if and only if it is upper almost $\mathcal{I}$-continuous.

Proof. Let $x \in X$ and $V$ be any regular open set of $Y$ containing $F(x)$. Since $(Y, \sigma)$ is extremally disconnected, is $V$ is regular closed and hence semiopen. By Theorem 3.8, there exists $U \in \mathcal{I} O(X, x)$ such that $F(U) \subset \mathrm{Cl}(V)=V$. Then $F$ is upper almost $\mathcal{I}$-continuous. Conversely, let $K$ be any regular closed subset of $Y$. Since $(Y, \sigma)$ is extremally disconnected, $K$ is also regular open and by Theorem 3.4 of [4], $F^{+}(K)$ is $\mathcal{I}$-open. By Theorem 3.8, $F$ is upper almost contra-I-continuous.

Theorem 3.14. Let $(Y, \sigma)$ be a extremally disconnected space. Then a multifunction $F:(X, \tau, \mathcal{I}) \rightarrow(Y, \sigma)$ is lower almost contra- $\mathcal{I}$-continuous if and only if $F$ is lower almost $\mathcal{I}$-continuous. 
Proof. The proof is similar to that of Theorem 3.13.

Theorem 3.15. The following statements are equivalent for a multifunction $F:(X, \tau, \mathcal{I}) \rightarrow(Y, \sigma)$ :

1. $F$ is upper (lower) almost contra- $\mathcal{I}$-continuous;

2. $F^{+}(\mathrm{Cl}(V))\left(F^{-}(\mathrm{Cl}(V))\right)$ is $\mathcal{I}$-open in $X$ for every $V \in S P O(Y)$;

3. $F^{+}(\mathrm{Cl}(V))\left(F^{-}(\mathrm{Cl}(V))\right)$ is $\mathcal{I}$-open in $X$ for every $V \in S O(Y)$;

4. $F^{-}(\operatorname{Int}(\mathrm{Cl}(V)))\left(F^{+}(\operatorname{Int}(\mathrm{Cl}(V)))\right)$ is $\mathcal{I}$-closed in $X$ for every $V \in P O(Y)$.

Proof. $(1) \Rightarrow(2)$ : Let $V$ be any semi-preopen set of $Y$. Since $\mathrm{Cl}(V) \in$ $R C(Y)$, by Theorem 3.8, $F^{-}(\mathrm{Cl}(V))$ is $\mathcal{I}$-open in $X$.

$(2) \Rightarrow(3)$ : This is obvious since $S O(Y) \subset S P O(Y)$.

$(3) \Rightarrow(4)$ : Let $V \in P O(Y)$. Then $Y \backslash \operatorname{Int}(\mathrm{Cl}(V))$ is regular closed and hence it is semiopen. Then, we have $X \backslash F^{-}(\operatorname{Int}(\mathrm{Cl}(V)))=F^{+}(Y \backslash \operatorname{Int}(\mathrm{Cl}(V)))$ $=F^{+}(\mathrm{Cl}(Y \backslash \operatorname{Int}(\mathrm{Cl}(V)))) \in \mathcal{I} O(X)$. Hence $F^{-}(\operatorname{Int}(\mathrm{Cl}(V))) \in \mathcal{I} C(X)$.

$(4) \Rightarrow(1)$ : Let $V$ be any regular open set of $Y$. This implies that $V \in$ $P O(Y)$ and hence $F^{-}(V)=F^{-}(\operatorname{Int}(\mathrm{Cl}(V)))$ is $\mathcal{I}$-closed in $X$. Hence $F$ is upper almost contra- $\mathcal{I}$-continuous.

The proof of the second case is similar.

Lemma 3.16. [19] For a subset $V$ of a topological space $(Y, \sigma)$, the following properties hold:

1. $\alpha \mathrm{Cl}(V)=\mathrm{Cl}(V)$ for every $V \in S P O(Y)$;

2. $p \mathrm{Cl}(V)=\mathrm{Cl}(V)$ for every $V \in S O(Y)$;

3. $s \mathrm{Cl}(V)=\operatorname{Int}(\mathrm{Cl}(V))$ for every $V \in P O(Y)$.

Corollary 3.17. The following statements are equivalent for a multifunction $F:(X, \tau, \mathcal{I}) \rightarrow(Y, \sigma)$ :

1. $F$ is upper (lower) almost contra- $\mathcal{I}$-continuous;

2. $F^{+}(\alpha \mathrm{Cl}(V))\left(F^{-}(\alpha \mathrm{Cl}(V))\right)$ is $\mathcal{I}$-open in $X$ for every $V \in S P O(Y)$;

3. $F^{+}(p \mathrm{Cl}(V))\left(F^{-}(p \mathrm{Cl}(V))\right)$ is $\mathcal{I}$-open in $X$ for every $V \in S O(Y)$;

4. $F^{-}(s \mathrm{Cl}(V))\left(F^{+}(s \mathrm{Cl}(V))\right)$ is $\mathcal{I}$-closed in $X$ for every $V \in P O(Y)$.

Proof. This is an immediate consequence of Theorem 3.15 and Lemma 3.16. 
Theorem 3.18. The following statements are equivalent for a multifunction $F:(X, \tau, \mathcal{I}) \rightarrow(Y, \sigma)$ :

1. $F$ is upper almost contra- $\mathcal{I}$-continuous;

2. $\mathcal{I} \mathrm{Cl}\left(F^{-}(V)\right) \subset F^{-}(\operatorname{Int}(\mathrm{Cl}(V))$ for every open subset $V$ of $Y$;

3. $\mathcal{I} \mathrm{Cl}\left(F^{-}(V)\right) \subset F^{-}(s \mathrm{Cl}(V))$ for every open subset $V$ of $Y$.

Proof. $(2) \Rightarrow(1)$ : Let $V \in R O(Y)$. Then $\mathcal{I} \mathrm{Cl}\left(F^{-}(V)\right) \subset F^{-}(\operatorname{Int}(\mathrm{Cl}(V))=$ $F^{-}(V)$. This implies that $F^{-}(A)$ is $\mathcal{I}$-closed and hence $F$ is upper almost contra- $\mathcal{I}$-continuous.

$(1) \Rightarrow(2)$ : Let $V$ be an open set. We have $\operatorname{Int}(\mathrm{Cl}(V)) \in R O(Y)$. By (1), $F^{-}\left(\operatorname{Int}(\mathrm{Cl}(V))\right.$ is $\mathcal{I}$-closed. Since $V \subset \operatorname{Int}(\mathrm{Cl}(A))$, then $F^{-}(A) \subset F^{-}(\operatorname{Int}(\mathrm{Cl}(A)))$. Thus, $\mathcal{I} \mathrm{Cl}\left(F^{-}(V)\right) \subset F^{-}(s \mathrm{Cl}(V))$.

$(2) \Leftrightarrow(3)$ : It follows from Lemma 3.16.

Theorem 3.19. The following statements are equivalent for a multifunction $F:(X, \tau, \mathcal{I}) \rightarrow(Y, \sigma)$ :

1. $F$ is lower almost contra- $\mathcal{I}$-continuous;

2. $\mathcal{I} \mathrm{Cl}\left(F^{+}(V)\right) \subset F^{+}(\operatorname{Int}(\mathrm{Cl}(V))$ for every open subset $V$ of $Y$;

3. $\mathcal{I} \mathrm{Cl}\left(F^{+}(V)\right) \subset F^{+}(s \mathrm{Cl}(V))$ for every open subset $V$ of $Y$.

Proof. The proof is similar to that of Theorem 3.18 .

Definition 3.20. A subset $A$ of a topological space $(X, \tau)$ is said to be:

1. $\alpha$-regular [12] if for each $a \in A$ and any open set $U$ containing $a$, there exists an open set $G$ of $X$ such that $a \in G \subset \mathrm{Cl}(G) \subset U$;

2. $\alpha$-paracompact [12] if every $X$-open cover $A$ has an $X$-open refinement which covers $A$ and is locally finite for each point of $X$.

Lemma 3.21. [12] If $A$ is an $\alpha$-regular $\alpha$-paracompact subset of a space $X$ and $U$ is an open neighborhood of $A$, then there exists an open set $G$ of $X$ such that $A \subset G \subset \mathrm{Cl}(G) \subset U$.

For a multifunction $F:(X, \tau) \rightarrow(Y, \sigma)$, a multifunction $\mathrm{Cl}(F): X \rightarrow Y$ is defined by $\mathrm{Cl}(F)(x)=\mathrm{Cl}(F(x))$ for each point $x \in X$. Similarly, we denote $s \mathrm{Cl}(F), p \mathrm{Cl}(F), \alpha \mathrm{Cl}(F), s p \mathrm{Cl}(F), \mathcal{I} \mathrm{Cl}(F)$. 
Lemma 3.22. [21] If $F:(X, \tau) \rightarrow(Y, \sigma)$ is a multifunction such that $F(x)$ is $\alpha$-paracompact $\alpha$-regular for each $x \in X$, then for each open set $V$ of $Y G^{+}(V)=F^{+}(V)$ and for each closed set $K$ of $Y G^{-}(K)=F^{-}(K)$, where $G$ denotes $\mathrm{Cl}(F)$, s $\mathrm{Cl}(F), p \mathrm{Cl}(F), \alpha \mathrm{Cl}(F)$, sp $\mathrm{Cl}(F), \mathcal{I} \mathrm{Cl}(F)$.

Lemma 3.23. [21] If $F:(X, \tau) \rightarrow(Y, \sigma)$ is a multifunction, then for each open set $V$ of $Y G^{-}(V)=F^{-}(V)$ and for each closed set $K$ of $Y G^{+}(K)=$ $F^{+}(K)$, where $G$ denotes $\mathrm{Cl}(F), s \mathrm{Cl}(F), p \mathrm{Cl}(F), \alpha \mathrm{Cl}(F)$, sp $\mathrm{Cl}(F), \mathcal{I} \mathrm{Cl}(F)$.

Theorem 3.24. A multifunction $F:(X, \tau, \mathcal{I}) \rightarrow(Y, \sigma)$ is upper almost contra- $\mathcal{I}$-continuous if and only if $G$ is upper almost contra- $\mathcal{I}$-continuous.

Proof. Let $K$ be a regular closed set of $Y$. By Theorem 3.8 and Lemma 3.23, $G^{+}(K)=F^{+}(K)$ is an $\mathcal{I}$-open set of $X$. Hence, $G$ is upper almost contra$\mathcal{I}$-continuous. Conversely, Let $K$ be a regular closed set of $Y$. By Theorem 3.8 and Lemma 3.23, $F^{+}(K)=G^{+}(K)$ is an $\mathcal{I}$-open set of $X$. Hence, $G$ is upper almost contra- $\mathcal{I}$-continuous.

Theorem 3.25. Let $F:(X, \tau, \mathcal{I}) \rightarrow(Y, \sigma)$ be a multifunction such that $F(x)$ is $\alpha$-regular $\alpha$-paracompact for each $x \in X$. Then $F$ is lower almost contra-I-continuous if and only if $G$ is lower almost contra- $\mathcal{I}$-continuous.

Proof. Let $K$ be a regular closed set of $Y$. By Theorem 3.9 and Lemma $3.22, G^{+}(K)=F^{+}(K)$ is an $\mathcal{I}$-open set of $X$. Hence, $G$ is upper almost contra$\mathcal{I}$-continuous. Conversely, Let $K$ be a regular closed set of $Y$. By Theorem 3.9 and Lemma 3.22, $F^{+}(K)=G^{+}(K)$ is an $\mathcal{I}$-open set of $X$. Hence, $G$ is upper almost contra- $\mathcal{I}$-continuous.

Theorem 3.26. Let $F:(X, \tau, \mathcal{I}) \rightarrow(Y, \sigma)$ be a multifunction and $U$ be an open subset of $X$. If $F$ is a lower (upper) almost contra-I-continuous, then $F_{\left.\right|_{U}}: U \rightarrow Y$ is lower (upper) almost contra-I-continuous.

Proof. Let $V$ be any regular closed set of $Y$. Let $x \in U$ and $x \in F_{\left.\right|_{U}}^{-}(V)$. Since $F$ is lower almost contra- $\mathcal{I}$-continuous multifunction, then there exists $G \in \mathcal{I} O(X, x)$ such that $G \subset F^{-}(V)$. Then by Lemma $2.2, x \in G \cap U \in \mathcal{I} O(A)$ and $G \cap U \subset F_{\left.\right|_{U}}^{-}(V)$. This shows that $F_{\left.\right|_{U}}$ is a lower almost contra- $\mathcal{I}$-continuous multifunction. The proof of the second case is similar.

Theorem 3.27. Let $\left\{U_{i}: i \in \Delta\right\}$ be an open cover of a space $X$. A multifunction $F:(X, \tau, \mathcal{I}) \rightarrow(Y, \sigma)$ is upper almost contra- $\mathcal{I}$-continuous if and only if the restriction $F_{\left.\right|_{i}}: U_{i} \rightarrow Y$ is upper almost contra- $\mathcal{I}$-continuous for each $i \in \Delta$. 
Proof. Suppose that $F$ is upper almost contra- $\mathcal{I}$-continuous multifunction. Let $i \in \Delta$ and $x \in U_{i}$ and $V$ be a regular closed set of $Y$ containing $F_{\mid U_{i}}(x)$. Since $F$ is upper almost contra- $\mathcal{I}$-continuous multifunction and $F(x)=F_{\mid U_{i}}(x)$, there exists $G \in \mathcal{I} O(X, x)$ such that $F(G) \subset V$. Set $U=G \cap U_{i}$, then $x \in U \in$ $\mathcal{I} O\left(U_{i}, x\right)$ and $F_{\mid U_{i}}(U)=F(U) \subset V$. Therefore, $F_{\mid U_{i}}$ is upper almost contra$\mathcal{I}$-continuous. Conversely, let $x \in X$ and $V \in R C(Y)$ containing $F(x)$. There exists $i \in \Delta$ such that $x \in U_{i}$. Since $F_{\mid U_{i}}$ is upper almost contra- $\mathcal{I}$-continuous and $F(x)=F_{\mid U_{i}}(x)$, there exists $U \in \mathcal{I} O\left(U_{i}, x\right)$ such that $F_{\mid U_{i}}(U) \subset V$. Then we have $U \in \mathcal{I} O(X, x)$ and $F(U) \subset V$. Therefore, $F$ is upper almost contra- $\mathcal{I}$ continuous.

\section{References}

[1] M. E. Abd El-Monsef, E. F. Lashien and A. A. Nasef, On $\mathcal{I}$-open sets and $\mathcal{I}$-continuous functions, Kyungpook Math. J., 32(1)(1992), 21-30.

[2] M. Akdag, On upper and lower $\mathcal{I}$-continuous multifunctions, Far East J. math. Sci., 25(1)(2007), 49-57.

[3] D. Andrijevic, Semi-preopen sets, Mat. Vesnik, 38(1986), 24-32.

[4] C. Arivazhai and N. Rajesh, Upper and lower almost $\mathcal{I}$-continuous functions (submitted).

[5] C. Arivazhai and N. Rajesh, On upper and lower contra-I-continuous multifunctions (submited).

[6] C. Arivazhai and N. Rajesh, Upper and lower weakly $\mathcal{I}$-continuous functions (submitted).

[7] T. Banzaru, Multifunctions and $M$-product spaces, Bull. Stin. Tech. Inst. Politech. Timisoara, Ser. Mat. Fiz. Mer. Teor. Apl., 17(31)(1972), 17-23.

[8] E. Ekici, On contra R-continuity and a weak form,Indian J. Math., 46(2-3)(2004), 267281.

[9] D. Jankovic and T. R. Hamlett, Compatible extension of ideals, Bull. U. M. I., 7(1992), 453-465.

[10] D. Jankovic and T. R. Hamlett, New Toplogies From Old Via Ideals, Amer. Math. Monthly, 97 (4) (1990), 295-310.

[11] J. E. Joseph and M. H. Kwack, On S-closed spaces, Proc. Amer. Math. Soc., 80(1980), 341-348.

[12] I. Kovacevic, Subsets and paracompactness, Univ. u. Novom Sadu, Zb. Rad. Prirod. Mat. Fac. Ser. Mat.,14(1984), 79-87.

[13] K. Kuratowski, Topology, Academic Press, New York, 1966.

[14] N. Levine, Semiopen sets and semicontinuity in topological spaces, Amer. Math. Monthly, 70(1963), 36-41.

[15] A. S. Mashhour, M. E. Abd El-Monsef and S. N. El-Deep, On precontinuous and weak precontinuous mappings, Proc. Math. Phys. Soc. Egypt, 53(1982), 47-53.

[16] R. A. Mahmoud and A. A. Nasef, Regularity and Normality via ideals, Bull. Malaysian Math. Sc. Soc., 24(2001), 129-136. 
[17] R. L. Newcomb, Topologies which are compact modulo an ideal, Ph.D. Thesis, University of California, USA(1967).

[18] O. Njastad, On some classes of nearly open sets, Pacific J. Math., 15(1965), 961-970.

[19] T. Noiri, On almost continuous functions, Indian J. Pure Appl. Math., 20(1989), 571-576.

[20] T. Noiri, Almost weakly continuous multifunctions, Demonstratio Math., 26(1993) 363380.

[21] T. Noiri and V. Popa, A unified theory of weak continuity for multifunctions, Stud. Cerc. St. Ser. Mat. Univ. Bacau, 16(2006), 167-200.

[22] T. Noiri and V. Popa, Almost weakly continuous multifunctions, Demonstratio Math.,26 (1993), 363-380.

[23] T. Noiri and V. Popa, A unified theory of almost continuity for multifunctions, Sci. Stud. Res. Ser. Math. Inform., 20(1) (2010),185-214.

[24] V. Popa, A note on weakly and almost continuous multifunctions, Univ, u Novom Sadu, Zb. Rad. Prirod-Mat. Fak. Ser. Mat., 21(1991),31-38.

[25] V. Popa, Weakly continuous multifunction, Boll. Un. Mat. Ital., (5) 15-A(1978),379-388.

[26] R. E. Simithson, Almost and weak continuity for multifunctions, Bull. Calcutta Math. Soc., 70(1978), 383-390.

[27] M. Stone, Applications of the theory of boolean rings to general topology, Trans. Amer. Math. Soc., 41(1937), 374-381.

[28] T. Thompson, S-closed spaces, Proc. Amer. Math. Soc., 60(1976), 335-338.

[29] R. Vaidyanathaswamy, The localisation theory in set topology, Proc. Indian Acad. Sci., 20(1945), 51-61. 
